\title{
ARTICLE
}

Epidemiology

\section{Statin use and risk of contralateral breast cancer: a nationwide cohort study}

\author{
Rikke Langballe ${ }^{1}$, Deirdre Cronin-Fenton ${ }^{2}$, Christian Dehlendorff ${ }^{1}$, Maj-Britt Jensen ${ }^{3}$, Bent Ejlertsen ${ }^{3,4}$, Michael Andersson ${ }^{4}$, \\ Søren Friis ${ }^{1,5}$ and Lene Mellemkjær ${ }^{1}$
}

BACKGROUND: Statins have demonstrated antineoplastic effects in breast cancer cell lines, particularly in oestrogen receptor (ER)negative cell lines. However, epidemiological studies have not supported a preventive effect of statin use against breast cancer. Therefore, we examined the association between statin use and contralateral breast cancer (CBC) risk among women with breast cancer.

METHODS: We identified 52,723 women with non-metastatic breast cancer during 1996-2012 from the Danish Breast Cancer Group database. We defined time-varying post-diagnosis statin use as minimum two prescriptions lagged by 1 year. Cox regression analyses were used to estimate hazard ratios (HRs) and 95\% confidence intervals (Cls) for CBC associated with statin use.

RESULTS: Statin use was associated with a lower $\mathrm{CBC}$ risk $(\mathrm{HR}=0.88 ; 95 \% \mathrm{Cl}=0.73-1.05)$. The inverse association was strongest for long-term use overall $(\mathrm{HR}=0.64 ; 95 \% \mathrm{Cl}=0.43-0.96)$, although the $\mathrm{HR}$ specifically for long-term consistent use and high-intensity use approached unity. Among ER-negative breast cancer patients, statin use was associated with a $\mathrm{CBC}$ risk reduction (HR $=0.67$; $95 \% \mathrm{Cl}=0.45-1.00$ ).

CONCLUSIONS: We found some indication that statins reduce the risk of CBC. Further evaluations are needed to disentangle the equivocal results for long-term use and to establish if ER-negative breast cancer patients may benefit most from statin use.

British Journal of Cancer (2018) 119:1297-1305; https://doi.org/10.1038/s41416-018-0252-1

\section{INTRODUCTION}

Statins reduce serum levels of cholesterol and prevent cardiovascular disease. ${ }^{1}$ Beneficial effects of statins against breast cancer have also been suggested in studies of breast cancer cell lines and animal models. ${ }^{2,3}$ These antineoplastic effects may be explained through blocking of the mevalonate pathway responsible for the synthesis of various products including cholesterol and isoprenoids that are involved in tumour initiation, growth and metastasis. ${ }^{4}$ Experimental studies have also suggested that the anti-neoplastic effects are limited to lipophilic statins, and notably pertain to oestrogen receptor (ER)-negative breast tumours, whereas hydrophilic statins have demonstrated no antineoplastic effects, regardless of ER status., ${ }^{2,5}$ Although the experimental findings are promising, epidemiologic studies of statin use and breast cancer risk have reported largely null associations, and a recent meta-analysis of 27 epidemiological studies and 9 randomised clinical trials (RCTs) concluded that statin use does not reduce the risk of breast cancer. ${ }^{6}$

A potential explanation of the null findings in studies of statin use and breast cancer risk may be that preventive effects of statins are attenuated in the general female population who have varying degrees of susceptibility to breast cancer. However, women who have developed breast cancer have proven susceptibility to the disease, and preventive measures against breast cancer may be more pronounced in such a high risk population. A study based on the Danish Breast Cancer Group (DBCG) and the nationwide Danish Prescription Registry reported a $46 \%$ reduced risk of contralateral breast cancer (CBC) associated with use of simvastatin, a lipophilic statin, up to 10 years after primary breast cancer diagnosis. ${ }^{7}$ However, CBC was not the primary outcome in that study, and no details on the association with $\mathrm{CBC}$ risk were presented.

We hypothesise that statin use reduces $\mathrm{CBC}$ risk among women with breast cancer, and that the protective effect is most pronounced in women with an ER-negative first breast cancer.

\section{METHODS}

Study population

We identified a cohort of patients with incident invasive breast cancer within the DBCG database. ${ }^{8}$ The DBCG database became nationwide in 1977 and includes detailed clinical information on the vast majority of patients (see Supplemental Material, Box 1 for additional details on the DBCG database and other nationwide registries used in the study).

The cohort comprised 64,914 women aged $\geq 20$ years with a first primary invasive breast cancer during 1996-2012. By starting the study cohort in 1996, we had at least one year of pre-diagnosis

\footnotetext{
${ }^{1}$ Danish Cancer Society Research Center, Copenhagen, Denmark; ${ }^{2}$ Department of Clinical Epidemiology, Aarhus University Hospital, Aarhus, Denmark; ${ }^{3}$ Danish Breast Cancer

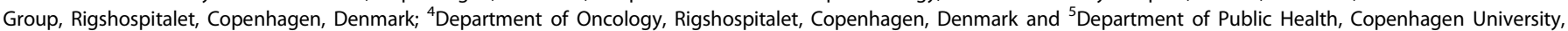
Copenhagen, Denmark

Correspondence: Lene Mellemkjær (lene@cancer.dk)
} 
Patients $\geq$ age 20 years with invasive breast cancer in the Danish Breast Cancer Group (DBCG) during 1996-2012:

$N=64,914$

Patients who did not receive surgery with curative intent: $N=3427$

Patients with

Unknown laterality: $N=838$

Synchronous laterality: $N=1447$

Patients with distant metastasis at first breast cancer: $N=498$

Patients with a previous cancer before first breast cancer diagnosis or 12 months after their first breast cancer diagnosis:

$N=3770$

Patients who received mastectomy of the contralateral breast $<12$ months following their first breast cancer: $N=748$

Patients who developed contralateral breast cancer $(\mathrm{CBC})<12$ months following their first breast cancer: $N=126$

Patients having a $\mathrm{CBC}$ in the Cancer Registry with similar laterality as the first breast cancer in DBCG (ipsilateral breast cancer) $<12$ months following their first breast cancer:

$N=108$

Patients who died $<12$ months following their first breast cancer:

$N=1188$

Patients who emigrated $<12$ months following their first breast cancer:

$N=41$

Final cohort

$N=52,723$

Fig. 1 Flow chart of study participants

statin use as the National Prescription Registry was first initiated in $1995 .{ }^{9}$ We excluded patients who did not undergo surgery with curative intent $(N=3427)$, had no record of laterality of the initial breast cancer $(N=838)$, or had synchronous $(N=1447)$ or metastatic disease $(N=498)$ at diagnosis. In addition, by linkage to the Danish Cancer Registry, ${ }^{10}$ we excluded patients who had a history of cancer (except non-melanoma skin cancer) prior to or within 1 year after the primary breast cancer $(N=3770)$. Finally, we excluded women who received mastectomy of the contralateral breast $(N=748)$ or developed cancer in the contralateral or same breast within the first year after the first breast cancer diagnosis $(N=234)$, and women who died or emigrated during this period $(N=1229)$. The final cohort comprised 52,723 breast cancer patients who were alive and at risk of CBC 1 year after the diagnosis (see flow chart in Fig. 1).

Assessment of statin use

From the National Prescription Registry, ${ }^{9}$ we retrieved information on statin prescriptions filled by the breast cancer patients between 1995 and 2013. We defined post-diagnosis statin use (see Supplemental Material, Table 1) as at least two prescriptions recorded on separate dates after the primary breast cancer diagnosis. Post-diagnosis statin use was lagged 1 year to allow a meaningful biological latency and to avoid reverse causation. ${ }^{11,12}$ 
Table 1. Baseline characteristics of 52,723 patients diagnosed with a first primary stage I-III breast cancer in Denmark during 1996-2012 according to statin use within the first year after cancer diagnosis

\begin{tabular}{|c|c|c|}
\hline \multirow[t]{3}{*}{ Characteristics } & \multicolumn{2}{|c|}{$\begin{array}{l}\text { Statin use within the year } \\
\text { following the first breast } \\
\text { cancer }^{\mathrm{a}}\end{array}$} \\
\hline & $\overline{Y e s}$ & No \\
\hline & Number (\%) & Number (\%) \\
\hline All & $5481(100)$ & $47,242(100)$ \\
\hline \multicolumn{3}{|l|}{ Age at first breast cancer diagnosis (years) ${ }^{b}$} \\
\hline $20-49$ & $133(2)$ & $10,454(22)$ \\
\hline $50-59$ & $991(18)$ & $13,457(28)$ \\
\hline $60-64$ & $1146(21)$ & $7078(15)$ \\
\hline $65-69$ & $1351(25)$ & $5997(13)$ \\
\hline $70-79$ & $1404(26)$ & $6854(15)$ \\
\hline$>=80$ & $456(8)$ & $3402(7)$ \\
\hline \multicolumn{3}{|c|}{ Menopausal status at first breast cancer diagnosis ${ }^{b}$} \\
\hline Premenopausal & $239(4)$ & $13,108(28)$ \\
\hline Postmenopausal & $5118(94)$ & $33,548(71)$ \\
\hline Unknown & $124(2)$ & $586(1)$ \\
\hline \multicolumn{3}{|l|}{ Calendar period of first breast cancer ${ }^{\mathrm{b}}$} \\
\hline $1996-2000$ & $235(4)$ & $13,416(28)$ \\
\hline $2001-2004$ & $651(12)$ & $10,944(23)$ \\
\hline $2005-2008$ & $1709(31)$ & $10,634(23)$ \\
\hline 2009-2012 & $2886(53)$ & $12,248(26)$ \\
\hline \multicolumn{3}{|l|}{ Histology of first breast cancer ${ }^{b}$} \\
\hline Ductal & $4299(78)$ & $37,577(79)$ \\
\hline Lobular & $533(10)$ & $5147(11)$ \\
\hline Other & $618(11)$ & $4194(9)$ \\
\hline Unknown & $31(1)$ & $324(1)$ \\
\hline \multicolumn{3}{|l|}{ Histology and grade of first breast cancer ${ }^{b}$} \\
\hline Ductal, Grade I & $1397(25)$ & $11,288(24)$ \\
\hline Ductal, Grade II-III & $2801(51)$ & $25,337(53)$ \\
\hline Other, Grade I-III/unknown & $1151(21)$ & $9341(20)$ \\
\hline Unknown & $132(2)$ & $1276(3)$ \\
\hline \multicolumn{3}{|l|}{ ER status at first breast cancer ${ }^{b}$} \\
\hline ER positive & $4565(83)$ & $37,028(78)$ \\
\hline ER negative & $844(15)$ & $8980(19)$ \\
\hline Unknown & $72(1)$ & $1234(3)$ \\
\hline \multicolumn{3}{|l|}{ Tumour size of first breast cancer ${ }^{b}$} \\
\hline$<=2 \mathrm{~cm}$ & $3413(62)$ & $27,667(59)$ \\
\hline $2.1-5 \mathrm{~cm}$ & $1834(34)$ & $16,726(35)$ \\
\hline$>=5.1 \mathrm{~cm}$ & $103(2)$ & $1618(3)$ \\
\hline Unknown & $131(2)$ & $1231(3)$ \\
\hline \multicolumn{3}{|l|}{ Lymph node status of first breast cancer ${ }^{b}$} \\
\hline Negative & $3110(57)$ & $24,359(52)$ \\
\hline Positive & $2166(39)$ & $20,932(44)$ \\
\hline Unknown & $205(4)$ & $1951(4)$ \\
\hline \multicolumn{3}{|l|}{ Treatment for first breast cancer $^{c}$} \\
\hline No treatment & $278(5)$ & $4189(9)$ \\
\hline Radiation treatment only & $822(15)$ & $6173(13)$ \\
\hline Chemotherapy only & $199(4)$ & $1988(4)$ \\
\hline Chemotherapy+radiation treatment & $466(9)$ & $4994(10)$ \\
\hline Endocrine treatment only & $784(14)$ & $4736(10)$ \\
\hline Endocrine treatment+radiation treatment & $1967(35)$ & $9744(21)$ \\
\hline
\end{tabular}

\begin{tabular}{|c|c|c|}
\hline \multirow[t]{3}{*}{ Characteristics } & \multicolumn{2}{|c|}{$\begin{array}{l}\text { Statin use within the year } \\
\text { following the first breast } \\
\text { cancer }{ }^{a}\end{array}$} \\
\hline & Yes & No \\
\hline & Number (\%) & Number (\%) \\
\hline Endocrine treatment+chemotherapy & $80(2)$ & $1360(3)$ \\
\hline $\begin{array}{l}\text { Endocrine treatment }+ \text { chemotherapy }+ \\
\text { radiation treatment }\end{array}$ & $518(10)$ & $7905(17)$ \\
\hline Unknown treatment & $367(6)$ & $6153(13)$ \\
\hline \multicolumn{3}{|l|}{ Comorbidity $^{d}$} \\
\hline Tobacco related diseases & $437(8)$ & $2320(5)$ \\
\hline Alcohol related diseases & $101(2)$ & $748(2)$ \\
\hline Diabetes & $928(17)$ & $1047(2)$ \\
\hline \multicolumn{3}{|l|}{ Other drug exposure } \\
\hline HRT, pre-diagnosis ${ }^{\mathrm{e}}$ & $1344(25)$ & $11,422(24)$ \\
\hline Aspirin, one-year post-diagnosis ${ }^{a}$ & $2133(39)$ & $3242(7)$ \\
\hline $\begin{array}{l}\text { Bisphosphonates, one-year post- } \\
\text { diagnosis }^{\mathrm{a}}\end{array}$ & $354(7)$ & $1418(3)$ \\
\hline Metformin, one-year post-diagnosis ${ }^{a}$ & $785(14)$ & $487(1)$ \\
\hline Digoxin, one-year post-diagnosis ${ }^{a}$ & $180(3)$ & $923(2)$ \\
\hline \multicolumn{3}{|c|}{ Educational level at first breast cancer diagnosis } \\
\hline Short (up to 9 years) & $1853(34)$ & $11,209(24)$ \\
\hline Medium (10-12 years) & $2632(48)$ & $21,296(45)$ \\
\hline High (>12 years) & $864(16)$ & $11,526(24)$ \\
\hline Unknown & $132(2)$ & $3211(7)$ \\
\hline \multicolumn{3}{|c|}{$\begin{array}{l}\text { CBC contralateral breast cancer, } E R \text { oestrogen receptor status, } H R T \text { hormone } \\
\text { replacement therapy } \\
\text { aDefined as } \geq 2 \text { prescriptions during the first year after the first breast } \\
\text { cancer diagnosis } \\
\text { bInformation retrieved from the Danish Breast Cancer Group } \\
\text { Intention to treat treatment retrieved from the Danish Breast Cancer } \\
\text { Group } \\
{ }^{d} \text { Defined as tobacco-related diseases, alcohol-related diseases and } \\
\text { diabetes mellitus } 10 \text { years prior to or one year after first breast cancer } \\
\text { diagnosis } \\
{ }^{e} \text { Defined as } \geq 2 \text { prescriptions within one year prior to the first breast cancer } \\
\text { diagnosis }\end{array}$} \\
\hline
\end{tabular}

In the main analysis, post-diagnosis statin use was modelled as a time-dependent variable. Thus, exposed person-time began from 1 year after the second prescription of statins, and persontime up to this point was considered as non-use. Moreover, we defined current statin use consecutively throughout follow-up as the period from filling a prescription plus the number of tablets and additional 90 days forward to allow minor non-compliance. Past-use of statins was defined as person-time among users not fulfilling the definition of exposure period for current use. Statin users were classified as consistent users until past use, if any, occurred where after they were classified as irregular users. Duration of statin use was defined as the interval between the first and latest prescription plus the number of tablets in the latest prescription and categorised as $<5$ years or $\geq 5$ years. Intensity of statin use (average daily statin dose) was consecutively estimated during follow-up as the cumulative number of DDDs divided by the number of days between the first and the latest statin prescription and classified as $<1 \mathrm{DDD} /$ day or $>=1 \mathrm{DDD} /$ day. Finally, we categorised subtypes of statins according to solubility (lipophilic/hydrophilic, see Table 2).

Follow-up for contralateral breast cancer

We used a previously established nationwide database on $\mathrm{CBCs}$ derived from the Danish Cancer Registry. ${ }^{10}$ According to coding 
Table 2. Hazard ratios (HRs) and $95 \%$ confidence intervals (Cls) of contralateral breast cancer (CBC) associated with post-diagnosis statin use, patterns of statin use and type of statin among 52,723 breast cancer patients during 1996-2012 in Denmark

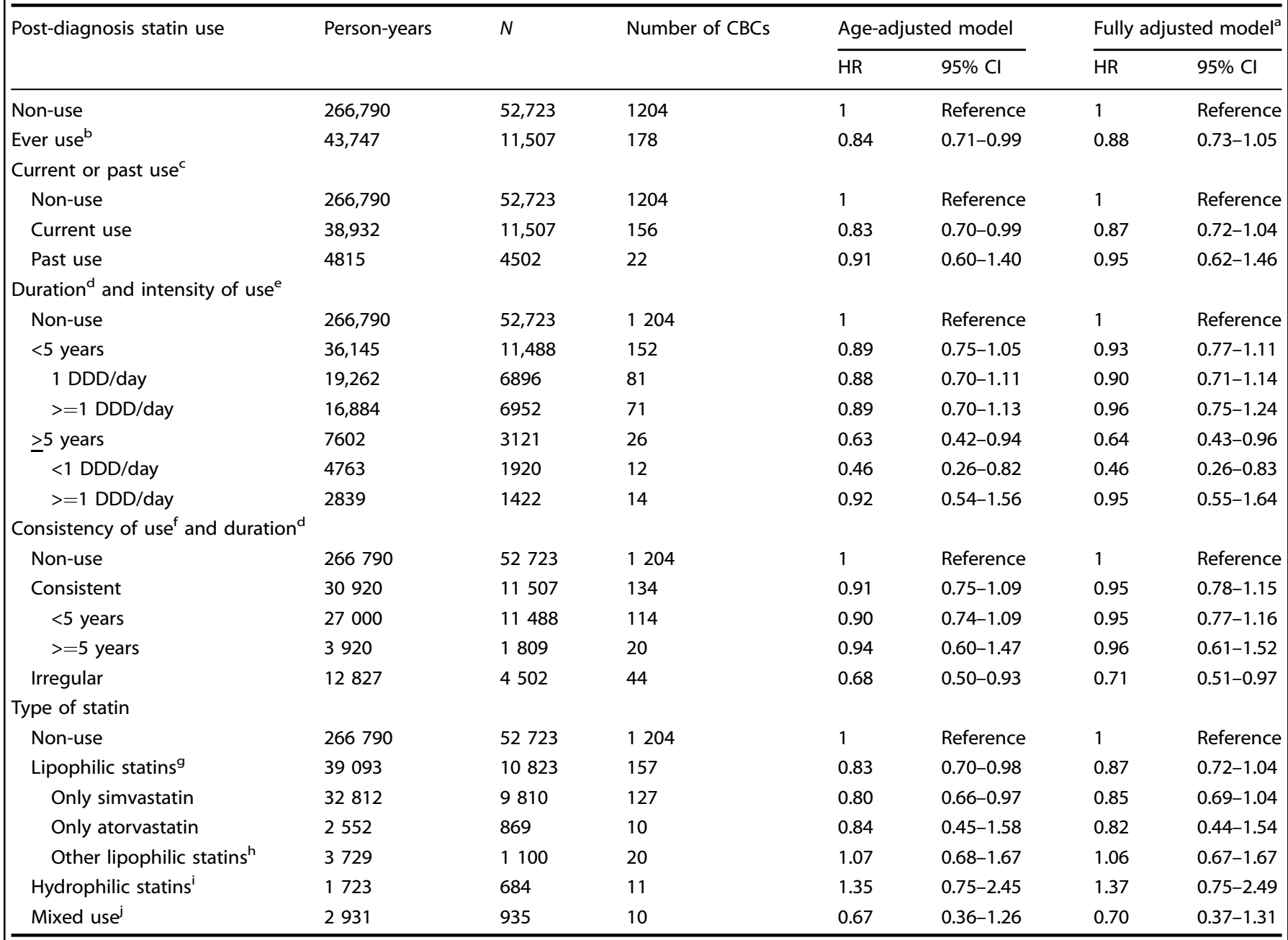

${ }^{a}$ Adjusted for age at first breast cancer, calendar-period at first breast cancer (1996-2000/2001-2004/2005-2008/2009-2012), lobular histology of first breast cancer (yes/no), treatment for first breast cancer (endocrine treatment only, chemotherapy only, radiation treatment only, endocrine treatment +chemotherapy, endocrine treatment+radiation treatment, chemotherapy+radiation treatment, endocrine treatment+chemotherapy+radiation treatment, no treatment and unknown treatment), pre-diagnosis exposure to hormone-replacement therapy (yes/no), time-dependent post-diagnosis exposure to aspirin, bisphosphonates, metformin and digoxin, alcohol-related conditions (yes/no), tobacco-related conditions (yes/no), diabetes mellitus (yes/no), educational level at first breast cancer diagnosis (short, medium, high, unknown)

${ }^{\mathrm{b}}$ Ever statin use was defined as $>=2$ prescriptions after the first breast cancer diagnosis

'Current use was defined as the period from redeeming a prescription plus the number of tablets and additional 90 days forward. Past use was defined as person-time among users not defined as current use

${ }^{\mathrm{d}}$ Duration of statin use was defined as the interval between the first and latest prescription plus the number of tablets in the latest prescription

e Intensity of use was defined as the cumulative number of defined daily doses (DDD) divided by duration of use in days

${ }^{\mathrm{f} C}$ Consistency of use was defined as current users with no prior periods as past users

'Lipophilic statin use was defined as users of simvastatin, atorvastatin, lovastatin, fluvastatin and cerivastatin only

${ }^{\mathrm{h}}$ Other lipophilic statin use was defined as users of lovastatin, fluvastatin and cerivastatin only

'Hydrophilic statins was defined as users of pravastatin and rosuvastatin only

${ }^{\mathrm{j}}$ Mixed use was defined as more than one type, e.g. simvastatin and atorvastatin

rules in the Cancer Registry, ${ }^{10}$ a second primary cancer occurring in paired organs with similar histology as the first primary tumour is not registered as an individual cancer record, but only labelled as 'bilateral'. Therefore, we obtained additional information on the CBC from the original notification forms to the Cancer Registry during 1978-2003, and from electronic records in the Danish Pathology Register during 2004-2013. ${ }^{13}$ Additional CBCs recorded in the DBCG only were added to the database. CBC with distant metastases at diagnosis was not counted as an outcome to limit possible misclassification of metastases from the first breast cancer $(N=58)$, but used as a censoring variable.

Follow-up for $\mathrm{CBC}$ started 1 year after the first breast cancer diagnosis (baseline) and continued until CBC, other cancer diagnosis (except non-melanoma skin cancer), ipsilateral breast cancer, prophylactic mastectomy of the contralateral breast, death, emigration or end of 2013, whichever came first. Contralateral mastectomy (see Supplemental Material, Table 1) was retrieved from the Danish National Patient Register. ${ }^{14}$ We 
Table 3. Hazard ratios (HRs) of contralateral breast cancer (CBC) and $95 \%$ confidence intervals (Cls) associated with post-diagnosis statin use according to oestrogen receptor (ER) status of first breast cancer (BC) diagnosis and CBC among 52,723 breast cancer patients during 1996-2012 in Denmark

\begin{tabular}{|c|c|c|c|c|c|c|c|}
\hline & Person-years & $N$ & Number of CBCs & \multicolumn{2}{|c|}{ Age-adjusted model } & \multicolumn{2}{|c|}{ Fully adjusted model ${ }^{a}$} \\
\hline \multicolumn{8}{|c|}{ ER status of first breast cancer } \\
\hline \multicolumn{8}{|l|}{ Positive } \\
\hline Non-use & 208,219 & 41,593 & 885 & 1 & Reference & 1 & Reference \\
\hline Ever statin use & 34,797 & 9331 & 144 & 0.89 & $0.74-1.06$ & 0.94 & $0.77-1.14$ \\
\hline Ever statin use & 7677 & 1903 & 28 & 0.65 & $0.44-0.97$ & 0.67 & $0.45-1.00$ \\
\hline \multicolumn{8}{|l|}{ Unknown } \\
\hline Non-use & 9313 & 1306 & 45 & 1 & Reference & 1 & Reference \\
\hline Ever statin use & 1273 & 273 & 6 & 0.16 & $0.06-0.38$ & 0.17 & $0.07-0.40$ \\
\hline \multicolumn{8}{|c|}{ ER status of contralateral breast cancer ${ }^{\mathrm{b}}$} \\
\hline \multicolumn{8}{|l|}{ Negative $^{d}$} \\
\hline Non-use & 266,790 & 52,723 & 214 & 1 & Reference & 1 & Reference \\
\hline Ever statin use & 43,747 & 11,507 & 26 & 0.91 & $0.59-1.38$ & 0.88 & $0.55-1.41$ \\
\hline \multicolumn{8}{|c|}{ ER status of first and second breast cancer } \\
\hline \multicolumn{8}{|c|}{ ER-positive first $B C$ and ER-positive $C B C^{C}$} \\
\hline Non-use & 208,219 & 41,593 & 652 & 1 & Reference & 1 & Reference \\
\hline Ever statin use & 34,797 & 9331 & 112 & 0.89 & $0.73-1.10$ & 0.93 & $0.74-1.16$ \\
\hline \multicolumn{8}{|c|}{ ER-positive first $B C$ and ER-negative $C B C^{d}$} \\
\hline Non-user & 208,219 & 41,593 & 120 & 1 & Reference & 1 & Reference \\
\hline Ever statin use & 34,797 & 9331 & 18 & 0.90 & $0.54-1.49$ & 0.91 & $0.51-1.61$ \\
\hline \multicolumn{8}{|c|}{ ER-negative first $B C$ and ER-positive $C B C^{C}$} \\
\hline
\end{tabular}

lagged the date of mastectomy by 30 days to ensure the procedure was prophylactic. Information on death and emigration was obtained from the Danish Civil Registration System. ${ }^{15}$

Definition of covariates

Information on potential confounding factors was retrieved from data available in the nationwide registries in Denmark. From DBCG, we included information on the primary breast cancer diagnosis: age (through a restricted cubic spline), date of breast cancer diagnosis (1996-2000/2001-2004/2005-2008/2009-2012), lobular histology (yes/no), and treatment (in eight categories; see footnote in Table 2). The DBCG guidelines define standard treatment modalities, which patients are allocated to according to age at diagnosis and tumour characteristics. ${ }^{16}$ We used the allocated treatment as adjustment variable in the analyses. Information on diabetes and alcohol- or tobacco-related diseases (yes/no) from 10 years prior to 1 year after the primary breast cancer was retrieved from the Danish National Patient Register. ${ }^{14}$ We also obtained information on use of prescription drugs from the National Prescription Registry, including pre-diagnosis hormone replacement therapy ( $>2$ prescriptions) and post-diagnosis use ( $>2$ prescriptions) of aspirin, bisphosphonates, metformin and digoxin. The Supplemental Material provides a detailed description of codes for drug exposure and covariates. From Statistics 
Table 4. Hazard ratios (HRs) of contralateral breast cancer (CBC) and $95 \%$ confidence intervals (Cls) associated with timing of statin use among 50,087 breast cancer patients during $1997-2012^{\mathrm{a}}$ in Denmark

\begin{tabular}{|c|c|c|c|c|c|c|c|}
\hline \multirow[t]{2}{*}{ Timing of statin use } & \multirow[t]{2}{*}{ Person-years } & \multirow[t]{2}{*}{$N$} & \multirow[t]{2}{*}{ Number of CBCs } & \multicolumn{2}{|c|}{ Age adjusted model } & \multicolumn{2}{|c|}{$\begin{array}{l}\text { Fully adjusted } \\
\text { model }^{\mathrm{b}}\end{array}$} \\
\hline & & & & $\overline{\mathrm{HR}}$ & $95 \% \mathrm{Cl}$ & $\overline{\mathrm{HR}}$ & $95 \% \mathrm{Cl}$ \\
\hline Never-use ${ }^{c}$ & 239,644 & 44,692 & 1063 & 1 & Reference & 1 & Reference \\
\hline Pre-diagnosis use $\mathrm{d}^{\mathrm{d}}$ & 3363 & 5395 & 11 & 0.95 & $0.52-1.75$ & 0.97 & $0.53-1.80$ \\
\hline Post-diagnosis use only ('new users') & 24,957 & 6339 & 107 & 0.85 & $0.69-1.05$ & 0.89 & $0.71-1.10$ \\
\hline Post- \& pre-diagnosis use ${ }^{\mathrm{e}}\left({ }^{\prime} c o n t i n u e r s '\right)^{\mathrm{e}}$ & 16,050 & 4691 & 56 & 0.79 & $0.60-1.04$ & 0.84 & $0.62-1.12$ \\
\hline \multicolumn{8}{|c|}{$\begin{array}{l}\text { This analysis was restricted to women diagnosed during 1997-2012 to allow pre-diagnosis exposure window of at least two years ( } \geq 2 \text { prescriptions) } \\
{ }^{b} \text { Adjusted for age at first breast cancer, calendar-period at first breast cancer (1996-2000/2001-2004/2005-2008/2009-2012), lobular histology of first breast } \\
\text { cancer (yes/no), treatment for first breast cancer (endocrine treatment only, chemotherapy only, radiation treatment only, endocrine treatment } \\
+ \text { chemotherapy, endocrine treatment+radiation treatment, chemotherapy+radiation treatment, endocrine treatment+chemotherapy+radiation treatment, } \\
\text { no treatment and unknown treatment), pre-diagnosis exposure to hormone-replacement therapy (yes/no), time-dependent post-diagnosis exposure to } \\
\text { aspirin, bisphosphonates, metformin and digoxin, alcohol-related conditions (yes/no), tobacco-related conditions (yes/no), diabetes mellitus (yes/no), } \\
\text { educational level at first breast cancer diagnosis (short, medium, higher, unknown) } \\
\text { cDiffers from the non-user category in Tables 2, } 3 \text { by not including person-years among pre-diagnosis users } \\
{ }^{d} \text { Defined as } \geq 2 \text { prescriptions within } 2 \text { years prior to the first breast cancer diagnosis }\end{array}$} \\
\hline
\end{tabular}

Denmark, ${ }^{17}$ we retrieved information on the highest achieved educational level at primary breast cancer diagnosis (short: up to 9 years; medium: 10-12 years; and high: $>12$ years).

\section{Statistical analyses}

We used cause-specific Cox regression models to estimate ageand fully adjusted hazard ratios (HRs) and 95\% confidence intervals (Cls) for risk of CBC associated with statin use. The fully adjusted model included the covariates defined above and the presented results in the text are from these models. Time since baseline was used as the underlying time scale. In the main analyses, we evaluated the association between post-diagnosis use of statins and patterns of statin use (current/past-use, duration, intensity and consistency) and type of statins as timevarying categorical variables compared to non-use. Effect measure modification was evaluated by stratified analyses according to information from DBCG on ER status of the primary breast cancer. Moreover, we analysed risk of ER-positive and ER-negative CBC associated with statin use by censoring at date of a non ERpositive and non ER-negative $\mathrm{CBC}$, respectively. We repeated the main analyses restricting statin exposure to (1) lipophilic statins only or (2) simvastatin only (see Supplemental Material for ATC codes). The proportional hazards assumption was evaluated by testing for trends in the scaled Schoenfeld residuals for ever use vs. non-use in the fully adjusted model.

In secondary analyses, we evaluated the influence of statin use prior to the first breast cancer on risk of $\mathrm{CBC}$, by using a timevarying exposure matrix combining pre- and post-diagnosis statin use, i.e., (1) no use before or after first breast cancer diagnosis (reference), (2) use before the diagnosis, (3) use solely after the first breast cancer diagnosis, and (4) use both before and after the diagnosis. This analysis was restricted to women diagnosed between 1997 and 2012 to allow a pre-diagnosis exposure window of at least 2 years.

We performed two pre-defined sensitivity analyses. First, we changed the definition of statin use to one prescription in the main analyses. Second, we examined the association between statin use ( $\geq 2$ prescriptions) and CBC risk using a fixed exposure period of 1 year following the first breast cancer diagnosis.

In post hoc sensitivity analyses, we also adjusted for menopausal status (premenopausal/postmenopausal) at first breast cancer diagnosis (women with unknown menopausal status $(N=710)$ were classified as premenopausal if younger than 50 years at diagnosis and postmenopausal if older than 50 years at diagnosis). In addition, we calculated a yearly updated propensity score using a logistic regression model to estimate the probability of being statin user during follow-up (time-varying) or the first year after breast cancer diagnosis (time-fixed). In the propensity model we included the same covariates as in the fully adjusted model as well as menopausal status, lymph node status (positive/negative/ unknown) and ER status (positive/negative/unknown) at first breast cancer diagnosis. ${ }^{18}$ In the Cox model, the propensity score was included through a restricted cubic spline together with age at first breast cancer and statin use (ever use/non-use).

Finally, we evaluated the potential impact of competing events in all analyses by estimating age- and fully adjusted HRs for the censoring criteria as one combined outcome (death, other cancer, ipsilateral breast cancer, distant disease at $C B C$ diagnosis and mastectomy of the contralateral breast).

All analyses were performed in $\mathrm{R}$ version $3.2 .3^{19}$ using the survival package, ${ }^{20}$ applying a $5 \%$ significance level and two-sided alternatives.

\section{RESULTS}

During the first year following the primary breast cancer, 5481 patients filled two or more statin prescriptions (Table 1). Statin users were more likely to be postmenopausal at first breast cancer diagnosis than non-users. The prevalence of statin use was higher among breast cancer patients in the later part of the study period, reflecting the increasing use of statins in the general population. ${ }^{21}$ Furthermore, statin users had a higher prevalence of diabetes, aspirin and metformin use, and shorter education compared with non-users.

During 310,537 person-years, 1382 breast cancer patients developed CBC. Overall, 11,507 patients filled two or more statin prescriptions and contributed person-years as statin users for a median of 3.3 years (interquartile range: 1.6-5.4 years). Postdiagnosis statin use was associated with a slightly reduced risk of $\mathrm{CBC}(\mathrm{HR}=0.88 ; 95 \% \mathrm{Cl}=0.73-1.05)$ (Table 2). A similar $\mathrm{HR}$ was seen with current statin use $(\mathrm{HR}=0.87 ; 95 \% \mathrm{Cl}=0.72-1.04)$, whereas the $\mathrm{HR}$ for $\mathrm{CBC}$ with past-use of statins approached unity $(\mathrm{HR}=0.95 ; 95 \% \mathrm{Cl}=0.62-1.46)$, however, numbers were small for past statin use.

We observed a reduced risk for $\mathrm{CBC}(\mathrm{HR}=0.64 ; 95 \% \mathrm{Cl}=$ $0.43-0.96)$ associated with post-diagnosis long-term use $(\geq 5$ 
years), whereas the association with shorter duration of statin use was close to unity ( $<5$ years) $(\mathrm{HR}=0.93 ; 95 \% \mathrm{Cl}=0.77-1.11)$ (Table 2). Stratification of duration of use according to intensity yielded a substantially reduced risk of $C B C$ associated with longterm ( $>5$ years), low intensity ( $<1 \mathrm{DDD} /$ day) statin use $(\mathrm{HR}=0.46$; $95 \% \overline{\mathrm{Cl}}=0.26-0.83)$, whereas no apparent inverse association emerged for long-term, high-intensity ( $>=1 \mathrm{DDD} /$ day) statin use $(\mathrm{HR}=0.95 ; 95 \% \mathrm{Cl}=0.55-1.64)$. In addition, we observed a reduced risk for $\mathrm{CBC}$ for irregular use of statins $(\mathrm{HR}=0.71 ; 95 \%$ $\mathrm{Cl}=0.51-0.97)$, but not for consistent use $(\mathrm{HR}=0.95 ; 95 \% \mathrm{Cl}=$ $0.78-1.15)$. In a post hoc analysis, we found no difference in $C B C$ risk when consistent statin use was stratified into short-term use ( $<5$ years: $\mathrm{HR}=0.95 ; 95 \% \mathrm{Cl}=0.77-1.16)$ and long-term use $(>5$ years: $\mathrm{HR}=0.96 ; 95 \% \mathrm{Cl}=0.61-1.52$ ). The majority of patients used the highly lipophilic statin, simvastatin (71\%) and a slightly reduced risk of $\mathrm{CBC}$ was seen for these users $(\mathrm{HR}=0.85 ; 95 \% \mathrm{Cl}=$ 0.69-1.04). Use of hydrophilic statins was associated with a statistically insignificant increased risk of CBC (HR: $1.37,95 \% \mathrm{Cl}=$ 0.75-2.49), however, use of these agents were rare and the number of $C B C$ was small. When restricting the analyses on patterns of use to lipophilic statins or simvastatin alone, the HRs remained unaltered (data not shown).

Among women with ER-negative primary breast cancer, we observed a reduced risk of $\mathrm{CBC}(\mathrm{HR}=0.67 ; 95 \% \mathrm{Cl}=0.45-1.00)$ associated with ever post-diagnosis statin use (Table 3 ), whereas no apparent inverse association was seen among women with ERpositive tumours ( $\mathrm{HR}=0.94 ; 95 \% \mathrm{Cl}=0.77-1.14)$. Among the statin users with ER-negative initial breast cancer, the HRs for ERpositive and ER-negative CBC were $0.64(95 \% \mathrm{Cl}=0.38-1.08)$ and 0.77 (95\% Cl=0.30-1.95), respectively. Restriction of these analyses to lipophilic use only yielded HRs similar to those in the main analyses (Supplemental Material, Table 2).

Among patients diagnosed between 1997 and 2012, prediagnosis statin use was associated with a neutral HR for CBC compared with never-use $(\mathrm{HR}=0.97 ; 95 \% \quad \mathrm{Cl}=0.53-1.80)$ (Table 4), whereas slightly reduced HRs were observed for new post-diagnosis users $(\mathrm{HR}=0.89 ; 95 \% \mathrm{Cl}=0.71-1.10)$ and for continuing users $(\mathrm{HR}=0.84 ; 95 \% \mathrm{Cl}=0.62-1.12)$.

The sensitivity analysis defining statin use as $\geq 1$ prescription showed no major change in the HR for overall post-diagnosis statin use ( $\mathrm{HR}=0.84 ; 95 \% \mathrm{Cl}=0.71-1.00)$. The analysis applying a fixed exposure period within the first year following the primary breast cancer yielded a HR of $0.76(95 \% \mathrm{Cl}=0.58-1.00)$.

In post hoc sensitivity analyses, we found no change in the risk estimates when adding menopausal status at first breast cancer diagnosis as a confounder. Finally, adjusting for the propensity of being statin user yielded virtually the same results as in the fully adjusted models.

The analyses for competing events showed largely the same pattern as for $\mathrm{CBC}$ risk estimates, i.e., use of statins was associated with a reduced risk of the competing events.

\section{DISCUSSION}

In this population-based cohort study of 52,723 breast cancer patients, we found a tendency towards a slightly reduced risk of CBC associated with ever and current statin use and a substantial reduction in $C B C$ risk with long-term statin use. Somewhat puzzling, we observed $C B C$ risk reductions associated with longterm statin use of low intensity or irregular use, but not with longterm high intensity or consistent statin use. A substantial reduction in $C B C$ risk with statin use was seen among patients with ER-negative primary breast cancer.

A Danish cohort study ${ }^{7}$ of 18,769 breast cancer patients from DBCG diagnosed during 1996-2003, thus overlapping with our study population, reported a reduction in $\mathrm{CBC}$ risk associated with the most commonly used lipophilic statin, simvastatin $(H R=0.54$; $95 \% \mathrm{Cl}=0.33-0.90)$. That association was based on 520 women with $\mathrm{CBC}$ within 10 years of their first breast cancer. A more recent, but smaller, cohort study from the United States ${ }^{22}$ had insufficient power to allow a meaningful conclusion on $\mathrm{CBC}$ risk. Our study was larger than previous studies and had more detailed information on statin type, timing and patterns of statin use, as well as on ER status of breast tumours.

Lipophilic statins have been suggested to possess the strongest antineoplastic effects due to their ability to cross cell membranes. ${ }^{2}$ Similar to our study, a meta-analysis of 10 studies evaluating breast cancer risk according to drug solubility has reported slight risk reductions associated with the highly lipophilic statin, simvastatin. ${ }^{6}$ Thus, so far there is a suggestion from this metaanalysis and the previous Danish study ${ }^{7}$ as well as our study that simvastatin use is associated with a decreased breast cancer risk.

Long-term use of statins ( $>5$ years) was suggested to be associated with a reduced risk of breast cancer in a meta-analysis based on 8 studies. $^{6}$ This previous study is compatible with our finding of a reduced $C B C$ risk associated with long-term use for more than 5 years. However, when we combined long-term use with the highest level of daily dose or with use without exposure gaps to identify the women who would be most heavily exposed to statins, we did not observe a pattern supporting a duration- or dose-response relationship. However, the results were based on relatively small numbers. Additional studies are needed to explore these equivocalities in our results, e.g. to investigate the dose level required to achieve antineoplastic effects of statins in humans.

Preventive measures for ER-negative breast cancer are highly warranted. ${ }^{23,24}$ Besides, women with ER-negative breast cancer are at increased risk of $\mathrm{CBC}$ compared with women diagnosed with the ER-positive phenotype, and in particular ER-negative $C B C .^{25,26}$ Previous epidemiological studies of either lipophilic statin use specifically ${ }^{27,28}$ or any statin type ${ }^{29-31}$ have found no evidence of a differential effect by ER status. Our study suggested a reduced risk of $C B C$ associated with statin use in patients with ER-negative primary breast cancer. This finding could imply an additional benefit of post-diagnosis statin use in addition to the growing evidence of reduced mortality ${ }^{32-35}$ and reduced risk of recurrences $3,7,34,36$ associated with statin use after breast cancer. Therefore, our results deserve to be evaluated further.

The strengths of our study included comprehensive nationwide data from a clinical database (DBCG), ensuring an unselected study population of breast cancer patients with high case validity. This was supplemented by high quality data from nationwide demographic and health registries ensuring virtually complete follow-up. Prescription data are not subject to recall bias, and statins are in Denmark available only by prescription. Large observational studies with long-term follow-up are valuable when investigating the influence of statin use on $C B C$ risk, given the difficulties to implement a randomised trial among breast cancer patients with sufficient power to detect rare and long-term adverse outcomes, such as CBCs. ${ }^{37}$

Our study also had some limitations. We had no information on compliance to statins and other drug use. We attempted to minimise exposure misclassification by defining drug use as at least two prescriptions filled on separate dates. In addition, adherence to statins in Denmark has been reported to be high $(>84 \%))^{38}$ Statin users were more likely to have shorter education and comorbidities than non-users, which do not support that the overall group of statin users in our study had a heathier lifestyle. Nevertheless, we cannot rule out that long-term low intensity statin use might reflect adherence to a healthier lifestyle. In all instances, the analyses were adjusted for education and comorbidity that are indicators of lifestyle. ${ }^{39-42}$ Our results suggested no major difference in overall risk estimates according to timing of statin use indicating that prevalent user bias may not be a major concern. Further analyses on patterns of statin use and ER status restricted to 'new users' are not likely to provide additional information on the issue of prevalent user bias due to 
limited statistical precision. Patients with recurrence following primary breast cancer may be more unlikely to be registered with a new primary tumour in the contralateral breast than those without recurrence. Since statin users tend to have fewer recurrences, this may have biased the association toward the null. In observational studies of statins and cancer risk reverse causality might impact results if patients undiagnosed with cancer experience weight loss and improved lipid profiles and consequently do not use statins. However, this is unlikely to have affected our results to any great extent because general or nonbreast symptoms are uncommon in patients with primary breast cancer. ${ }^{43}$ In addition, we censored patients with distant metastases at $C B C$ diagnosis and other cancer diagnoses for whom disease symptoms potentially could have affected their use of statins. Finally, we cannot exclude the possibility of important residual confounding from unmeasured variables associated with both statin use and $\mathrm{CBC}$ risk.

In conclusion, we found some support of a reduced risk of $\mathrm{CBC}$ associated with statin use among breast cancer patients. Further studies are required to clarify the equivocal findings according to pattern of statin use, notably for long-term use, and to determine if statin use could be particularly useful for women with breast cancer of the ER-negative phenotype.

\section{ACKNOWLEDGEMENTS}

This work was funded by the Scientific Committee of the Danish Cancer Society (grant number R90-A5792-14-S2); Henrik Henriksens Fond; Holger og Inez Petersens Mindelegat and Fru Astrid Thaysens Legat for Lægevidenskabelig Grundforskning.

\section{AUTHOR CONTRIBUTIONS}

L.M., S.F., D.C.F., M.A., B.E., and M.J. participated in writing the study protocol. M.J. and B.E. provided data for the study. L.M., S.F., C.D., D.C.F. and R.L. wrote the data analyses plan. L.M., S.F., C.D. and D.C.F. provided supervision. C.D. performed all statistics. R.L. drafted and coordinated the revision of the manuscript. All authors significantly contributed to the critical revision of the manuscript.

\section{ADDITIONAL INFORMATION}

Supplementary information is available for this paper at https://doi.org/10.1038/ s41416-018-0252-1.

Competing interests: Bent Ejlertsen has received research grants to his institution from Novartis, Roche and NanoString outside the submitted work. Maj-Britt Jensen has received personal fees from AstraZeneca and travel support from AstraZeneca, Novartis and Celgene outside the submitted work. Lene Mellemkjær has an immediate family member employed at Novo Nordisk, and has an immediate family member who owns stocks in Novo Nordisk. The remaining authors declare no competing interests.

Ethics approval: The study was approved by the Danish Data Protection Agency (J. no. 2012-41-0284). In Denmark, approvals from Ethical Committees are not required for register-based studies that do not involve biological samples.

NoteThis work is published under the standard license to publish agreement. After 12 months the work will become freely available and the license terms will switch to a Creative Commons Attribution 4.0 International (CC BY 4.0).

\section{REFERENCES}

1. Chou, R., Dana, T., Blazina, I., Daeges, M. \& Jeanne, T. L. Statins for prevention of cardiovascular disease in adults: evidence report and systematic review for the US Preventive Services Task Force. JAMA 316, 2008-2024 (2016).

2. Campbell, M. J. et al. Breast cancer growth prevention by statins. Cancer Res. 66, 8707-8714 (2006)

3. Ahern, T. P., Lash, T. L., Damkier, P., Christiansen, P. M. \& Cronin-Fenton, D. P. Statins and breast cancer prognosis: evidence and opportunities. Lancet Oncol. 15, 461-468 (2014).

4. Borgquist, S., Bjarnadottir, O., Kimbung, S. \& Ahern, T. P. Statins - a role in breast cancer therapy? J. Intern. Med. https://doi.org/10.1111/joim.12806 (2018).
5. Vinayak, S. \& Kurian, A. W. Statins may reduce breast cancer risk, particularly hormone receptor-negative disease. Curr. Breast Cancer Rep. 1, 148-156 (2009).

6. Islam, M. M. et al. Exploring association between statin use and breast cancer risk: an updated meta-analysis. Arch. Gynecol. Obstet. 296, 1043-1053 (2017).

7. Ahern, T. P. et al. Statin prescriptions and breast cancer recurrence risk: a Danish nationwide prospective cohort study. J. Natl. Cancer Inst. 103, 1461-1468 (2011).

8. Christiansen, P., Ejlertsen, B., Jensen, M. B. \& Mouridsen, H. Danish Breast Cancer Cooperative Group. Clin. Epidemiol. 8, 445-449 (2016).

9. Pottegard, A. et al. Data Resource Profile: The Danish National Prescription Registry. Int. J. Epidemiol. 46, 798-798 (2017).

10. Gjerstorff, M. L. The Danish Cancer Registry. Scand. J. Public Health 39, 42-45 (2011).

11. Chubak, J., Boudreau, D. M., Wirtz, H. S., McKnight, B. \& Weiss, N. S. Threats to validity of nonrandomized studies of postdiagnosis exposures on cancer recurrence and survival. J. Natl. Cancer Inst. 105, 1456-1462 (2013).

12. Tamim, H., Monfared, A. A. \& LeLorier, J. Application of lag-time into exposure definitions to control for protopathic bias. Pharmacoepidemiol. Drug Saf. 16, 250-258 (2007)

13. Bjerregaard, B. \& Larsen, O. B. The Danish Pathology Register. Scand. J. Public Health 39, 72-74 (2011).

14. Lynge, E., Sandegaard, J. L. \& Rebolj, M. The Danish National Patient Register. Scand. J. Public Health 39, 30-33 (2011).

15. Pedersen, C. B. The Danish Civil Registration System. Scand. J. Public Health 39, 22-25 (2011).

16. Moller, S. et al. Theclinical database and the treatment guidelines of the Danish Breast Cancer Cooperative Group (DBCG); its 30-years experience and future promise. Acta Oncol. 47, 506-524 (2008).

17. Jensen, V. M. \& Rasmussen, A. W. Danish Education Registers. Scand. J. Public Health 39, 91-94 (2011).

18. Brookhart, M. A. et al. Variable selection for propensity score models. Am. J. Epidemiol. 163, 1149-1156 (2006).

19. R Foundation for Statistical Computing. R: A language and environment for statistical computing. https://www.R-project.org/. Accessed 05/2017.

20. Therneau, T. M., \& Grambsch, P. M. Modeling Survival Data: Extending the Cox Model. (Springer: New York, 2000).

21. Schmidt, M., Hallas, J., Laursen, M. \& Friis, S. Data Resource Profile: Danish online drug use statistics (MEDSTAT). Int. J. Epidemiol. 45, 1401-1402 (2016).

22. Boudreau, D. M. et al. Comparative safety of cardiovascular medication use and breast cancer outcomes among women with early stage breast cancer. Breast Cancer Res. Treat. 144, 405-416 (2014).

23. Cuzick, J. et al. Preventive therapy for breast cancer: a consensus statement. Lancet Oncol. 12, 496-503 (2011).

24. Li, Y. \& Brown, P. H. Translational approaches for the prevention of estrogen receptor-negative breast cancer. Eur. J. Cancer Prev. 16, 203-215 (2007).

25. Reiner, A. S. et al. Hormone receptor status of a first primary breast cancer predicts contralateral breast cancer risk in the WECARE study population. Breast Cancer Res. 19, 83 (2017).

26. Kurian, A. W. et al. Second primary breast cancer occurrence according to hormone receptor status. J. Natl. Cancer Inst. 101, 1058-1065 (2009).

27. Borgquist, S. et al. Statin use and breast cancer risk in the nurses' health study. Cancer Epidemiol. Biomark. Prev. 25, 201-206 (2016).

28. Woditschka, S., Habel, L. A., Udaltsova, N., Friedman, G. D. \& Sieh, W. Lipophilic statin use and risk of breast cancer subtypes. Cancer Epidemiol. Biomark. Prev. 19, 2479-2487 (2010).

29. Boudreau, D. M. et al. Statin use and breast cancer risk in a large populationbased setting. Cancer Epidemiol. Biomark. Prev. 16, 416-421 (2007).

30. Cauley, J. A. et al. Statin use and breast cancer: prospective results from the Women's Health Initiative. J. Natl. Cancer Inst. 98, 700-707 (2006).

31. Eliassen, A. H., Colditz, G. A., Rosner, B., Willett, W. C. \& Hankinson, S. E. Serum lipids, lipid-lowering drugs, and the risk of breast cancer. Arch. Intern. Med. 165, 2264-2271 (2005)

32. Zhong, S. et al. Statin use and mortality in cancer patients: systematic review and meta-analysis of observational studies. Cancer Treat. Rev. 41, 554-567 (2015)

33. Wu, Q. J. et al. Statin use and breast cancer survival and risk: a systematic review and meta-analysis. Oncotarget 6, 42988-43004 (2015).

34. Manthravadi, S., Shrestha, A. \& Madhusudhana, S. Impact of statin use on cancer recurrence and mortality in breast cancer: a systematic review and meta-analysis. Int. J. Cancer 139, 1281-1288 (2016).

35. Smith, A., Murphy, L., Zgaga, L., Barron, T. I. \& Bennett, K. Pre-diagnostic statin use, lymph node status and mortality in women with stages I-III breast cancer. Br. J. Cancer 117, 588-596 (2017).

36. Borgquist, S. et al. Cholesterol, cholesterol-lowering medication use, and breast cancer outcome in the BIG 1-98 study. J. Clin. Oncol. 35, 1179-1188 (2017). 
37. Bonovas, S., Lytras, T. \& Sitaras, N. M. Statin use and breast cancer: do we need more evidence and what should this be? Expert Opin. Drug Saf. 13, 271-275 (2014).

38. Svensson, E., Nielsen, R. B., Hasvold, P., Aarskog, P. \& Thomsen, R. W. Statin prescription patterns, adherence, and attainment of cholesterol treatment goals in routine clinical care: a Danish population-based study. Clin. Epidemiol. 7 213-223 (2015)

39. Groth, M. V., Fagt, S. \& Brondsted, L. Social determinants of dietary habits in Denmark. Eur. J. Clin. Nutr. 55, 959-966 (2001).

40. Molsted, S., Johnsen, N. F. \& Snorgaard, O. Trends in leisure time physical activity, smoking, body mass index and alcohol consumption in Danish adults with and without diabetes: a repeat cross-sectional national survey covering the years 2000 to 2010. Diabetes Res. Clin. Pract. 105, 217-222 (2014).

41. Schnohr, C. et al. Does educational level influence the effects of smoking, alcohol, physical activity, and obesity on mortality? A prospective population study. Scand. J. Public Health 32, 250-256 (2004).

42. Hansen, H., Johnsen, N. F. \& Molsted, S. Time trends in leisure time physical activity, smoking, alcohol consumption and body mass index in Danish adults with and without COPD. BMC Pulm. Med. 16, 110 (2016).

43. Koo, M. M. et al. Typical and atypical presenting symptoms of breast cancer and their associations with diagnostic intervals: evidence from a national audit of cancer diagnosis. Cancer Epidemiol. 48, 140-146 (2017). 